\title{
Caracteres agronómicos en el cultivo de espárrago de diferentes edades y manejos
}

\author{
Pablo Diego Asprelli(1), Fernando Sebastián López Anido ${ }^{(1)}$ y Enrique Luis Cointry ${ }^{(1)}$
}

(1)Universidad Nacional de Rosario, Facultad de Ciencias Agrarias, Cátedra de Genética, Parque “J. F. Villarino”, CC14 (ZAA2125S), Zavalla,
Santa Fe, Argentina. E-mail: pasprel@fcagr.unr.edu.ar, felopez@fcagr.unr.edu.ar, ecointry@fcagr.unr.edu.ar

Resumen - El objetivo del trabajo fue evaluar el efecto de la edad de la planta y la respuesta a los manejos en 11 híbridos de espárrago (Asparagus officinalis L.). Se utilizó un diseño en bloques completos aleatorios con tres repeticiones de 20 plantas por parcela, con tres y cuatro años de edad, según dos sistemas de manejo: con surcos alomados para la producción de espárrago blanco y surcos sin alomar para espárrago verde. Con el manejo para espárrago blanco se obtuvieron mayores valores promedio para días a brotación, rendimiento de mercado, rendimiento total, número de turiones y peso promedio del turión. Sin embargo, la tasa de incremento del primer al segundo año de cosecha para rendimiento de mercado y rendimiento total resultó superior en el manejo verde debido a una tasa de incremento superior para número de turiones. Para días a brotación y peso promedio del turión, la respuesta debida al efecto del manejo y de la edad del cultivo fue similar, mientras que días al $50 \%$ de parcela brotada se vio afectada principalmente por la edad de la planta.

Términos para indexación: Asparagus officinalis, precocidad, uniformidad de brotación, productividad, tasa de incremento.

\section{Agronomic characters in asparagus of different ages and crop systems}

\begin{abstract}
Age and crop system effects on agronomic response were evaluated in 11 hybrids of Asparagus officinalis L. The experimental design was a complete randomized block with three replicates of 20 plants of 3 or 4 years old, conducted either in mounded soil over plants for blanched asparagus, or in raised beds without ridging for green asparagus. The highest mean values were obtained under the blanched production for days to harvest, market yield, total yield, spears number and the spear mean weight. However, under the green production, the rate of increment from first to second year was superior for market yield and total yield due to an augmented rate of increment of spears number. Type and year of production effects were similar for days to first harvest and spears mean weight, meanwhile days to $50 \%$ of sprout plot was affected mainly by the year of production.
\end{abstract}

Index terms: Asparagus officinalis, earliness, sprout uniformity, productivity, rate of increment.

\section{Introducción}

El espárrago (Asparagus officinalis L.) es originario de la región oriental del Mediterráneo y Asia menor y crece en climas templados y subtropicales, siendo la única especie de su género cultivada como hortaliza (Ornstrup, 1997). La planta de espárrago presenta una porción subterránea conocida como araña que consiste en un rizoma expandido lateralmente y poco profundo que posee yemas en su extremo apical, raíces carnosas de almacenamiento y raíces laterales de absorción (Ornstrup, 1997). El crecimiento de la araña ocurre cuando las yemas periféricas originan nuevos grupos de gemación para la próxima generación de la planta (Tiedjens, 1926).

$\mathrm{Al}$ aumentar la temperatura hacia finales del invierno emergen del rizoma los brotes jóvenes llamados turiones, que son la parte comestible de la planta (Ornstrup, 1997), dependiendo su color de la variedad y del sistema de manejo (Barón \& Vallejo, 1990). Los turiones que se desarrollan luego de la etapa de cosecha (primaveraverano) forman el follaje conocido como helecho, el cual comienza a senescer en el otoño. El nivel de sacarosa de la raíz aumenta durante el período de dormancia invernal y puede servir como señal de inducción de las yemas cuando la temperatura aumenta (Drost, 1997).

El espárrago tiene un período promedio de cosecha de 10 años y llega al máximo de producción a los cuatro o cinco años. Una elevada productividad es esencial para tener un buen retorno de la inversión, teniendo en cuenta que el precio de turiones grandes es mayor que el de los pequeños, registrándose los precios más elevados al inicio de la primavera cuando comienzan a comercializarse los primeros volúmenes (Ellison, 1986). 
El mejoramiento del cultivo apunta a mayores producciones, mejor calidad y adaptabilidad climática (Reuther, 1984). Las altas temperaturas causan la apertura prematura de los extremos de los turiones, haciendo que se ramifiquen a baja altura, volviéndose no comerciables (Ellison, 1986), por ello la precocidad es de gran importancia para el consumo en fresco. Además, la posibilidad de disponer de materiales de espárrago de brotación uniforme permitiría lograr una producción concentrada, reduciendo el período de colecta con la concomitante disminución de costos. Desde el punto de vista productivo, los caracteres más importantes en el mejoramiento del espárrago son el número y el peso promedio de los turiones ya que son las variables determinantes del rendimiento (Gatti et al., 2000). Ellison (1986), trabajando con las mismas plantas durante tres años consecutivos, observó que los rendimientos máximos provinieron de plantas que tenían muchos turiones grandes, y que las plantas con muchos turiones pequeños o con pocos turiones grandes no tuvieron altos rendimientos. El tamaño de la yema está positivamente correlacionado con el tamaño del turión (Nichols \& Woolley, 1985). La dimensión de los brotes que la planta puede producir estaría gobernada por procesos genéticamente determinados, aunque sería afectada por el tipo de manejo y el nivel de reservas (Blasberg, 1932).

Si bien existe información sobre el efecto de las prácticas culturales sobre la productividad del cultivo del espárrago, aún no se conoce completamente como interaccionan con la fisiología del mismo (Drost, 1997). Un carácter que se observa en dos ambientes puede estar determinado en parte por mecanismos fisiológicos diferentes y consecuentemente los genes requeridos son también hasta cierto punto distintos (Falconer \& Mackay, 1996). La respuesta a diferentes climas y sistemas de manejo son características importantes en un híbrido de espárrago, y al mismo tiempo difíciles de predecir (Ellison, 1986).

El objetivo de este trabajo fue conocer la influencia del sistema de manejo y de la edad del cultivo sobre caracteres agronómicos en híbridos clonales de espárrago.

\section{Material y Métodos}

La experiencia se realizó en el campo experimental "J. F. Villarino" de la Facultad de Ciencias Agrarias de la Universidad Nacional de Rosario, ubicado en la localidad de Zavalla, provincia de Santa Fe, Argentina $\left(33^{\circ} 1^{\prime} \mathrm{S}, 60^{\circ} 53^{\prime} \mathrm{O}, 50 \mathrm{~m}\right.$ sobre el nivel del mar).
En 1999 fueron implantadas arañas de un año de edad de 11 híbridos clonales (HEZ 264, HEZ 274, HEZ 284, HEZ 294, HEZ 304, HEZ 314, HEZ 324, HEZ 334, HEZ 344, HEZ 354 y HEZ 364) derivados de nuestro programa de mejora de espárrago en un diseño en bloques completos aleatorios con tres repeticiones de 20 plantas por parcela. La distancia de plantación fue $2.1 \mathrm{~m}$ entre surcos y $0.4 \mathrm{~m}$ entre plantas. Los materiales fueron analizados durante las campañas 2000 y 2001 cuando las plantas tenían tres y cuatro años de edad, respectivamente, y fueron conducidos según dos sistemas de manejo: con surcos alomados para la producción de espárrago blanco y surcos sin alomar para espárrago verde.

Las observaciones fueron hechas sobre parcelas individuales, evaluándose los días a brotación (DAB) como el número de días transcurridos desde el 13 de abril (fecha coincidente con el corte de la masa aérea) hasta la cosecha del primer turión que es representativa de la precocidad; días al $50 \%$ de parcela brotada (D50\%) como el número de días desde la cosecha del primer turión hasta que el $50 \%$ de las plantas de una parcela iniciaron su producción, que es indicativa de la uniformidad de brotación de las plantas; rendimiento de mercado (RM) como el peso total en gramos de los turiones que presentaron color uniforme, sección circular, extremo compacto, y un diámetro igual o mayor a $10 \mathrm{~mm}$ medidos en la base del turión para el sistema de manejo de espárrago verde y de $12 \mathrm{~mm}$ para espárrago blanco tomados en la base del tercio superior del turión; rendimiento total (RT) como el peso en gramos de los turiones cosechados; número de turiones cosechados (NT) y peso medio del turión (PT) obtenido por medio del cociente RT/NT.

En ambas campañas los turiones fueron cosechados durante seis semanas contadas a partir del momento en que se alcanzó el 50\% de brotación de cada parcela con el fin de uniformar las observaciones de las variables productivas ya que períodos largos de cosecha llevan a un desdoblamiento excesivo de carbohidratos, disminuyendo los niveles de reservas en la raíz, reduciendo el desempeño de la planta a largo plazo. Para evitar tal efecto, la duración de la cosecha fue similar en las dos primeras campañas productivas bajo ambos sistemas de manejo Previo a ser pesados, los turiones cosechados fueron cortados a $15 \mathrm{~cm}$ de longitud. Se realizó el registro de las temperaturas mínima, media y máxima mensual en el período de inducción-producción comprendido entre los meses de julio y diciembre. 
Para establecer el comportamiento de los caracteres evaluados bajo ambos sistemas de manejo en función de la edad del cultivo se realizó un análisis de variancia anidado por el procedimiento GLM del programa SAS (SAS Institute Inc., 1982), siendo las fuentes de variación los materiales híbridos, los años de cosecha, el tipo de manejo dentro de los años de evaluación y la interacción entre los genotipos híbridos y los manejos dentro de cada año. Se compararon los valores promedios a través de la prueba de Duncan.

Con el fin de determinar la influencia del sistema de manejo sobre el desarrollo del cultivo al aumentar la edad de las plantas, se calcularon las tasas de incremento proporcional del primer al segundo año productivo como $\left[\left(\mathrm{X}_{2001}-\mathrm{X}_{2000}\right) / \mathrm{X}_{2000}\right] .100$, siendo $\mathrm{X}$ el valor medio observado de cada una de las variables evaluadas en cada año de cosecha para cada sistema de manejo. La comparación se efectuó por medio de la prueba de chicuadrado (Snedecor, 1956).

\section{Resultados y Discusión}

Los materiales híbridos mostraron un comportamiento diferencial para las diferentes variables, estando su comportamiento afectado por el sistema de manejo, la edad del cultivo o bien por ambos factores según el carácter que se considere (Cuadro 1). A su vez, el análisis de los materiales, discriminando entre los sistemas de manejo y las campañas productivas (Cuadro 2), permitió obtener una mejor descripción del comportamiento de los caracteres evaluados.

Para días a brotación, no se observaron diferencias entre los materiales híbridos, pero la diferencia encontrada entre las campañas productivas (Cuadro 1) se debió a que en promedio las parcelas comenzaron a producir siete días más tarde en el segundo año de producción que en el primero, independientemente del sistema de manejo (Cuadro 2). Este efecto pudo ser originado por una menor temperatura ambiental promedio en el año 2001 durante el período de acumulación térmica para la inducción, comprendido entre los meses de julio y septiembre (Figura 1), ya que la tasa de inducción y crecimiento de las yemas responden directamente al aumento de temperatura (Dean, 1999). También se encontraron diferencias significativas para D50\% (Cuadro 1), ya que los materiales fueron de brotación más uniforme en la segunda campaña productiva que en la primera (Cuadro 2).

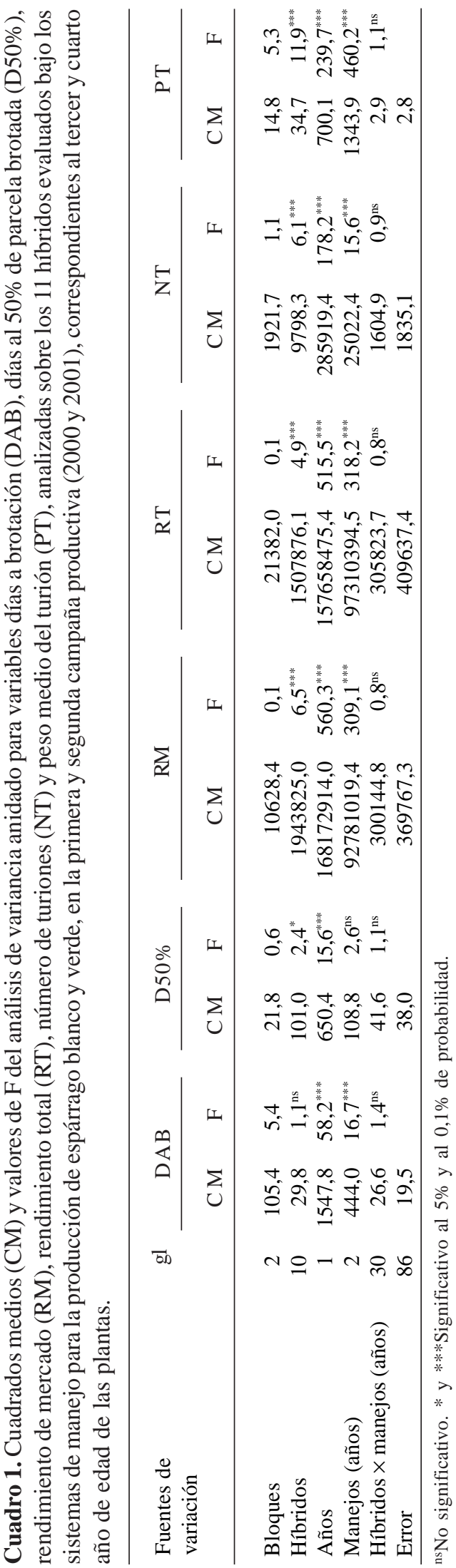

Pesq. agropec. bras., Brasília, v.40, n.1, p.47-52, jan. 2005 
Al retrasarse el inicio de la cosecha en el año 2001 por efecto de la menor temperatura ambiente durante los meses de julio y agosto (Figura 1), el incremento en la uniformidad de brotación bajo ambos sistemas de manejo se debió a que el crecimiento de las yemas fue más concentrado a comienzos de la primavera cuando los materiales se vieron expuestos a la temperatura mínima para lograr la inducción. Un aumento de temperatura induciría una actividad metabólica superior, promoviendo el desdoblamiento más rápido de reservas, y al poner una mayor cantidad de azúcares a disposición de las yemas en un período más corto se incrementaría notablemente la tasa de emisión de turiones cuando las plantas brotan más tardíamente (Drost, 1997).

Respecto a las variables productivas, se encontraron diferencias significativas tanto entre los materiales híbridos como entre los períodos evaluados para RM, RT, NT y PT (Cuadro 1), resultando, en todos los materiales, mayores en las plantas de cuatro años de edad (año 2001) que en las de tres (año 2000) (Cuadro 2). Gatti et al. (2000) demostraron que a igual período de cosecha el rendimiento aumenta progresivamente con la edad del cultivo debido al incremento del número de turiones

Cuadro 2. Valores promedio y errores estándar para días a brotación (DAB), días al 50\% de parcela brotada (D50\%), rendimiento de mercado (RM), rendimiento total (RT), número de turiones (NT) y peso medio del turión (PT) analizadas bajo los sistemas de manejo para espárrago blanco y verde en plantas de tres y cuatro años de edad, correspondiente al primer y segundo año productivo $(2000 \text { y } 2001)^{(1)}$.

\begin{tabular}{|c|c|c|c|c|}
\hline \multirow[t]{2}{*}{ Variable } & \multirow[t]{2}{*}{ Año } & \multicolumn{2}{|c|}{ Tipo de manejo } & \multirow[t]{2}{*}{ Promedio de años } \\
\hline & & Blanco & Verde & \\
\hline \multirow[t]{2}{*}{ DAB (dias) } & 2000 & $163,1 \pm 1,0 \mathrm{a}$ & $158,1 \pm 0,8 b$ & $160,6 \pm 0,7 \mathrm{~B}$ \\
\hline & 2001 & $170,2 \pm 0,8 \mathrm{a}$ & $164,7 \pm 0,7 \mathrm{~b}$ & $167,5 \pm 0,6 \mathrm{~A}$ \\
\hline \multirow[t]{2}{*}{ D50\% (dias) } & 2000 & $23,1 \pm 1,2 \mathrm{a}$ & $20,9 \pm 1,2 \mathrm{a}$ & $22,0 \pm 0,8 \mathrm{~A}$ \\
\hline & 2001 & $16,1 \pm 0,8 \mathrm{a}$ & $19,0 \pm 1,3 \mathrm{a}$ & $17,6 \pm 0,8 \mathrm{~B}$ \\
\hline \multirow[t]{2}{*}{ RM (g/parcela) } & 2000 & $1979,7 \pm 105,2 \mathrm{a}$ & $516,3 \pm 625,0 \mathrm{~b}$ & $1248,0 \pm 109,1 \mathrm{~B}$ \\
\hline & 2001 & $5014,1 \pm 181,6 \mathrm{a}$ & $1996,8 \pm 95,9 b$ & $3505,5 \pm 213,1 \mathrm{~A}$ \\
\hline \multirow[t]{2}{*}{ RT (g/parcela) } & 2000 & $2837,2 \pm 117,1 \mathrm{a}$ & $1110,0 \pm 78,3 b$ & $1973,6 \pm 127,9 \mathrm{~B}$ \\
\hline & 2001 & $5643,6 \pm 167,1 \mathrm{a}$ & $2675,1 \pm 92,7 b$ & $4159,4 \pm 207,1 \mathrm{~A}$ \\
\hline \multirow[t]{2}{*}{ NT (g/parcela) } & 2000 & $185,1 \pm 8,5 \mathrm{a}$ & $135,7 \pm 7,2 b$ & $160,4 \pm 6,3 \mathrm{~B}$ \\
\hline & 2001 & $265,7 \pm 9,0 \mathrm{a}$ & $241,3 \pm 9,3 b$ & $253,5 \pm 6,6 \mathrm{~A}$ \\
\hline \multirow[t]{2}{*}{ PT (g/turión) } & 2000 & $15,6 \pm 0,4 \mathrm{a}$ & $8,1 \pm 0,3 b$ & $11,8 \pm 0,5 \mathrm{~B}$ \\
\hline & 2001 & $21,6 \pm 0,6 a$ & $11,3 \pm 0,3 b$ & $16,5 \pm 0,7 \mathrm{~A}$ \\
\hline
\end{tabular}

(1) Promedios seguidos de letras diferentes, minúsculas en las filas y mayúsculas en la columna, indican diferencias significativas en la prueba de Duncan $(\mathrm{p}<0,05)$
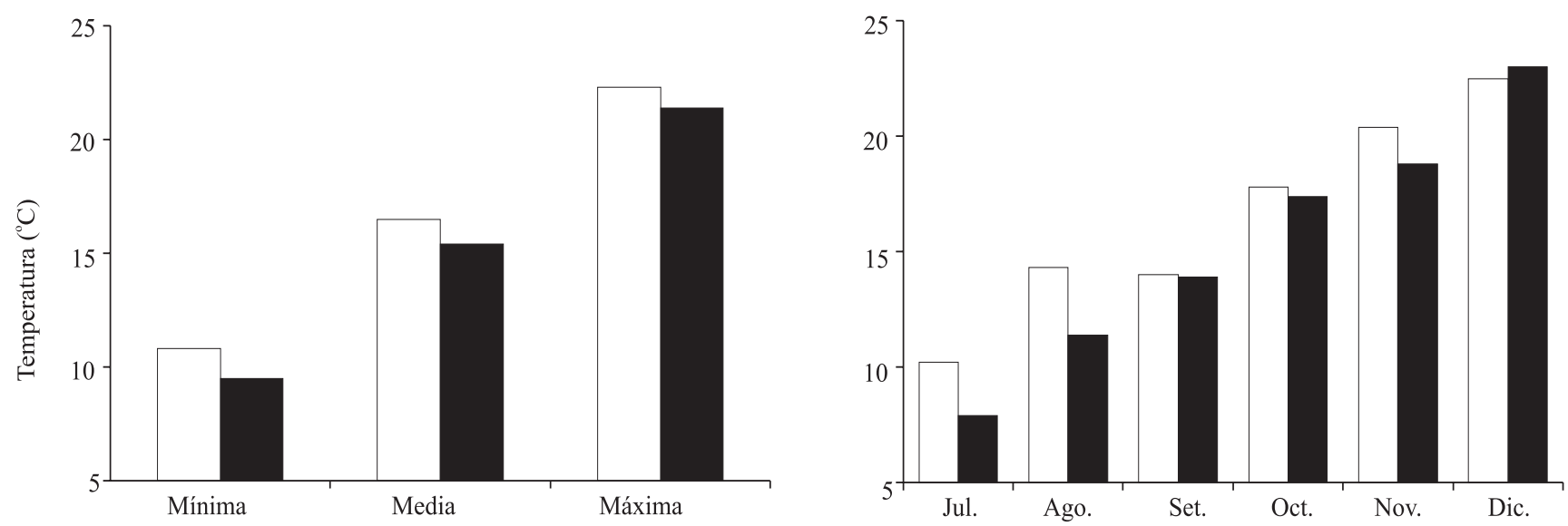

Figura 1. Temperaturas mínimas, medias y máximas y temperaturas medias mensuales del segundo semestre de los años $2000(\square)$ y 2001 ( $\square$ ) registradas en el campo experimental. 
producidos, ya que el rendimiento está determinado por el número y el tamaño de las yemas subterráneas diferenciadas en la etapa anterior de crecimiento (Tiejdens, 1926).

El peso medio del turión mostró diferencias significativas entre años independientemente del manejo. Esto se debería a que la porción perenne de la planta produciría año tras año un número mayor de yemas que dan origen a turiones de tamaño comercial y no por un aumento del diámetro de las yemas en sí mismas, ya que el grosor de los turiones es un carácter que queda definido en el primer año productivo para toda la vida útil de la esparraguera (Cointry et al., 2000).

En lo referente a la influencia del sistema de manejo, los materiales mostraron diferencias significativas para días a brotación en cada período de cosecha (Cuadro 1), observándose que las parcelas entraron en producción cinco días antes en el sistema de manejo verde que en el blanco en los dos años de evaluación (Cuadro 2). Esto se debería a que en principio el crecimiento del turión está en función de la temperatura del suelo y al aumentar la profundidad de plantación disminuye el índice de desarrollo de las yemas, por lo que en el sistema de manejo blanco se retarda la producción (Lindgren, 1990). En el manejo verde la inducción de las yemas está determinada por el aumento de temperatura del suelo (Drost, 1997), mientras que el crecimiento de los turiones depende fundamentalmente de la temperatura del aire (Krarup \& Krarup, 1987), ya que la mayor velocidad de crecimiento se ubica por debajo del extremo apical del turión (Keuls \& Post, 1957). Con relación a D50\%, la influencia del sistema de manejo en cada año de evaluación resultó no significativa, ya que entre los sistemas de manejo blanco y verde hubo una diferencia de dos días en el año 2000 y de tres días en el 2001 (Cuadro 2).

Se encontraron diferencias significativas para RM, RT, NT y PT (Cuadro 1), siendo que a igual densidad de plantación los valores promedio de estas variables fueron mayores en el sistema de manejo blanco (Cuadro 2). Al igual que lo observado por Knaflewski (1990), cuando los híbridos fueron conducidos para la producción de espárrago blanco produjeron turiones de peso medio mayor que cuando fueron conducidos para la producción de espárrago verde. Al aumentar la profundidad de plantación el crecimiento de los turiones se retrasa ya que las yemas quedan expuestas a temperaturas menores durante el período de brotación, lo que promueve un mayor diámetro (Lindgren, 1990). Krarup \& Krarup (1987) informaron que a igualdad de diámetro los turiones blancos pesaron más que los verdes debido a una mayor densidad específica. Los turiones que crecen en la oscuridad tienen mayor contenido de sólidos solubles que los turiones verdes (Ketsa \& Uthaiburt, 1987), menor contenido de proteínas, ácido ascórbico y compuestos fenólicos (Shewfelt \& Mhor, 1960). Esto parece indicar que el turión durante su crecimiento sería capaz de responder anatómica y metabólicamente a las condiciones ambientales inmediatas, dirigiendo su fisiología de acuerdo a si crece en condiciones de oscuridad o de luminosidad.

$\mathrm{Al}$ analizar los incrementos proporcionales de los valores promedio de las variables productivas de la segunda campaña respecto a la primera, se observó una respuesta diferencial en función del sistema de manejo, siendo éstos superiores en el manejo verde para RM, RT y NT, y similar para PT (Cuadro 3). Si bien los valores promedio de estas variables resultaron mayores en plantas de cuatro años de edad y en el sistema de manejo blanco, la mayor tasa de incremento para RM y RT bajo el sistema de manejo verde habría sido originada por un mayor incremento proporcional del número de turiones producidos.

A pesar que el rendimiento depende del número y tamaño de los turiones, el número de turiones emitidos sería la componente más importante del rendimiento que lo afectaría de manera directa, tal como observaran Ito \& Currens (1964). Este hecho tendría su origen en los

Cuadro 3. Tasas de incremento porcentual del primer al segundo año de cosecha correspondientes al tercer y cuarto año de edad de las plantas para rendimiento de mercado, rendimiento total, número de turiones y peso medio del turión en los sistemas de producción para espárrago blanco y verde ${ }^{(1)}$.

\begin{tabular}{lcccc}
\hline Tipo de manejo & \multicolumn{2}{c}{ Tasa de incremento porcentual } \\
\cline { 2 - 5 } & $\begin{array}{c}\text { Rendimiento } \\
\text { de mercado }\end{array}$ & $\begin{array}{c}\text { Rendimiento } \\
\text { total }\end{array}$ & $\begin{array}{c}\text { Número } \\
\text { de turiones }\end{array}$ & $\begin{array}{c}\text { Peso promedio } \\
\text { de turión }\end{array}$ \\
\hline Blanco & $153,3 \mathrm{a}$ & $98,9 \mathrm{a}$ & $43,5 \mathrm{a}$ & $38,8 \mathrm{a}$ \\
Verde & $286,8 \mathrm{~b}$ & $141,1 \mathrm{~b}$ & $77,8 \mathrm{~b}$ & $39,1 \mathrm{a}$ \\
\hline
\end{tabular}

${ }^{(1)}$ Valores porcentuales seguidos de letras diferentes indican diferencias significativas en la prueba de chi-cuadrado $(\mathrm{p}<0,05)$. 
procesos fisiológicos que regulan el crecimiento vegetativo, ya que el diámetro del turión está relacionado con el tamaño de la araña, el número de yemas y la cantidad de carbohidratos disponibles (Blasberg, 1932). En el caso del espárrago, la cantidad de fotoasimilados almacenados en las raíces actuaría como recurso limitante en la determinación del número y el diámetro de los turiones de modo que un mayor número implica un menor diámetro y viceversa, existiendo una compensación entre ellos (Cointry et al., 2000).

De mantenerse la tendencia descripta, en el tercer año de cosecha (quinto año de la planta) los híbridos producirían un mayor número de turiones si son manejados para la producción de espárrago verde. Sin embargo, el rendimiento no alcanzaría igual volumen que cuando los materiales se manejen para la producción de espárrago blanco, debido a la diferencia de peso medio de los turiones generada por los distintos sistemas de manejo.

\section{Conclusiones}

1. La precocidad se ve influida tanto por el sistema de manejo como por las condiciones ambientales del período de inducción.

2. El tamaño de los turiones depende del sistema de manejo, mostrando un aumento similar en los dos tipos de manejo respecto a la edad del cultivo.

3. La respuesta diferencial al manejo para rendimiento de mercado y rendimiento total se debe a diferencias en la tasa de incremento del número de turiones emitidos.

\section{Agradecimientos}

A la Agencia Nacional de Promoción Científica y Tecnológica, por los fondos aportados a través del PICT № 08-07333, para esta investigación.

\section{Referencias}

BARÓN, C.G.; VALLEJO, H. Asparagus whitening using black polyethilene. Asparagus Research Newsletter, v.8, p.13-23, 1990.

BLASBERG, C.H. Phases of the anatomy of asparagus officinalis. Botanical Gazette, v.94, p.206-214, 1932.

COINTRY, E.L.; LÓPEZ ANIDO, F.S.; GATTI, I.; CRAVERO, V.P.; FIRPO, I.T.; GARCÍA, S.M. Early selection of elite plants in asparagus. Bragantia, v.59, p.21-26, 2000.
DEAN, B.B. The effect of temperature on asparagus spear growth and correlation of heat units accumulated in the field with spear yield. Acta Horticulturae, v.479, p.289-295, 1999.

DROST, D.T. Asparagus. In: WIEN, H.C. (Ed.). The physiology of vegetable crops. Cambridge: CAB Internacional, 1997. p.621-649.

ELLISON, J.H. Asparagus breeding. In: BASSET, M.J. (Ed.). Breeding Vegetables Crops. Westport: AVI, 1986. p.521-569.

FALCONER, D.S.; MACKAY, T.F.C. Introduction to quantitative genetics. $4^{\text {th }}$ ed., London: longman, 1966. 64p.

GATTI, I.; CRAVERO, V.P.; LÓPEZANIDO, F.S.; COINTRY, E.L. Evaluación de siete poblaciones de espárrago (Asparagus officinalis L.). Pesquisa Agropecuária Brasileira, v.35, p.1151-1157, 2000.

ITO, P.J.; CURRENCE, T.M. Inbreeding and heterosis in asparagus. Proceeding of the American Society for Horticultural Science, v.86, p.338-346, 1965.

KETSA, S.; UTHAIBURT, J. Chemical composition of white and green spears of asparagus (Asparagus officinalis L.). Asian Food Journal, v.3, p.77-78, 1987.

KEULS, M.; POST, J.J. Invloed van de temperatuur op de groi van asperges. Overdruk uit de Mededelingen Directeur Tuinbouw, v.19, p.827-845, 1957.

KNAFLEWSKI, M. Comparison of the suitability of asparagus cultivars for blanched and green spear production. Acta Horticulturae, v.271, p.191-196, 1990.

KRARUP, A.H.; KRARUP, J.P.L. Rendimiento de espárragos verdes y blancos bajo dos modalidades de cosecha. Agro Sur, v.15, p.47-53, 1987.

LINDGREN, D.T. Influence of planting deep and interval to initial harvest on yield and plant response of asparagus. HortScience, v. 25, p.754-756, 1990.

NICHOLS, M.A.; WOOLLEY, D. Growth studies with asparagus. In: INTERNATIONAL ASPARAGUS SYMPOSIUM, 6., Proceedings. Ontario: University of Guelph, 1985. p.287-297.

ORNSTRUP, O. Biotechnological methods in asparagus breeding. Asparagus Research Newsletter, v.14, p.1-25, 1997.

REUTHER, G. Asparagus. In: SHARP, W.R.; EVANS, D.A.; AMMIRATO, P.V.; YAMADA, Y. (Ed.). Handbook of plant cell culture. New York: MacMillan Publishing Company, 1984. p.211-242.

SAS INSTITUTE (Cary, Estados Unidos). SAS user's guide: statistics. Cary, 1982. 584 p.

SHEWFELT, A.L.; MOHR, W.P. Effect of spear length, spear portion and harvest date on the composition and quality of asparagus. Canadian Journal of Plant Science, v.40, p.371-374, 1960.

SNEDECOR, G.W. Statistical methods. $4^{\text {th }}$ ed. Ames: Iowa State University Press, 1956. 626p.

TIEDJENS, V.A. Some observations on root and crown bud formation in Asparagus officinalis. Proceeding of the American Society for Horticultural Science, v.23, p.189-195, 1926.

$\overline{\text { Recibido el } 23 \text { de marzo de } 2004 \text { y aceptado el } 2 \text { de septiembre de } 2004}$ 